\title{
Swimming turned technology teaching and training guide the optimization of diagnosis
}

\author{
Chao Chen ${ }^{1,}$ a and Bing Zhang 2 , b \\ ${ }^{1}$ Institute of Physical Education, Jilin Normal University, Siping 136000, China \\ 2Institute of Physical Education, Huanggang Normal University, Huangzhou 438000, China
}

\begin{abstract}
Swimming is a physical and technical project, reasonable and scientific technical characteristics are a powerful guarantee to improve performance. Athletics swimming movement is mainly composed of starts and turns, on the way to swim, to edge, and at the end of the sprint composed of five parts such as technology, because of athletes in using the same technique will have a lot of individual differences, this paper analyzes the process of the diagnosis of competitive swimming technology optimization to improve the athletes technical rationality and scientific nature makes the corresponding theoretical basis. Data analysis in this paper, through a large number of game to perplex a competitive swimmer turn, Chinese technology system research, in order to China's competitive swimming training scientific reasonable Suggestions.
\end{abstract}

\section{Introduction}

Swimming event has a profound history, starting from international swimming federation was founded in 1908 till now, through above one hundred years development, such event has become a sports event of greater impacts in Olympic Games, and is also one of important events that every country snatches gold in Olympic Games. Modern swimming event competition becomes increasingly fierce, winning or losing in competition only has 0.01 second difference, as long as any one technical motion has unscientific factors in whole competitive swimming, it can generate huge impacts on competition performance ranking. Swimming event result is mainly up to physical ability and technique two aspects, physical ability is basis, but technique is guarantee. Nowadays, swimming event rapidly develops; short distance swimming event world record almost gets closer to human body extreme ability. Domestic and foreign countries have carried on widely and deepen analysis and research on competitive swimming technical features. The paper carries on theoretical and data analysis of competitive swimming technique diagnosis optimization and turn start techniques, in the hope of providing certain theoretical basis for Chinese swimming training [1-3].

On the basis of formers research theories, the paper studies on competitive swimming technique diagnosis optimization and turn start technique, in the hope of making contributions to Chinese competitive swimming operation event undertakings.

a,b Corresponding author: a 398704001@qq.com;

\section{Competitive swimming movement technical analysis}

Movement technique is a process; it has features of quantity and quality. Quantity feature includes kinematics and dynamics features, it can make quantitative testing, analysis and evaluation by corresponding instrument equipment and methods ways; quality feature includes rhythm, accuracy and range as well as other features, which can use coaches and athletes to make qualitative analysis and evaluation on them [4].Whether swimming technical analysis can correct, really reflect athlete technical status and level is foundation of diagnosing athletes techniques, is also important basis to propose improvement and perfection suggestions as well as countermeasures [4-6].

Though competitive swimming techniques mainly include starting, turning, on passage swimming, reaching to wall and sprint as well as others five techniques, athletes have many differences in the same technical movements, it has certain individual specialty. Just in view of technical perspective, partial technique and technique details tend to decide competitive swimming level high-low. By making technical analysis of every athlete, targeted at athlete each technical link, movement structure, stroking instant features, make quantitative analysis and unscrambling from dynamics and kinematics orientations, and further correct judge key technical problem that constraints high level athlete to further improve competitive levels. In the following, we study from competitive swimming technical

\footnotetext{
b tiyuxi@qq.com
} 
diagnosis optimization and starting turning technique two aspects.

Competitive swimming technical diagnoses

Swimming athletes' technical diagnosis is key factor to optimize athlete technical movement and improve athlete training efficiency. In technical problem diagnosis, we should clearly distinguish and grasp that athlete technical level further improvement restriction is physical ability factor or technical problem, only get objective 、 correct conclusion and carry on targeted effective training then can really give competitive swimming athletes maximum potential into really play. To arrive at technical diagnosis reliability and accuracy target, it should combine with athlete technical analysis report, multi-media technical analysis information, by deepen exchanging between coaches and athletes, it further improves recognition on competitive swimming techniques, and carries on research analysis from world top swimming athletes technical features, world top swimming athletes technical database( including competition video, multi-perspective technical simulation animation ) and world swimming event development trend the three main aspects to define national excellent swimming athletes technical optimization scheme, and establish a set of complete strong targeted and high efficiency training method [7].

\subsection{Competitive swimming technical diagnosis analysis}

Evidence for making qualitative evaluation and analysis of athletes' movement technical process is coach, athlete and relative scientific research staff recognition level on competitive swimming techniques, and meanwhile also play decisive roles in diagnosing swimming techniques. Recognition on a sports event features is a process from the shallower to the deeper, is up to two aspects, on one hand is coach self recognition ability, more important aspect is science and technology as well as research method progress and development. Emergence of new theory can open up new perspective for project features recognition, appearance of new technique and new methods can promote recognition degree deepening. With times development, competitive swimming is constantly promoting, it surely will appear more scientific competitive swimming technical theory and technical training method.We can make research analysis of new technical theories and training methods to deepen exchange with athletes and coaches, and then promote their recognition on competitive swimming technique, resonate and make popularization and application in daily technical training and practice.

In Figure 1, A: athlete competitive swimming technical analysis report's: competition technical and tactical indicators analysis report's: in-depth exchange with coaches, athletes and enhance competitive swimming technical recognition; D: swimming event development trend and world top swimming athlete technical features; E: world top swimming athlete techniques database; F: Define national excellent swimming athlete technical optimization plan.

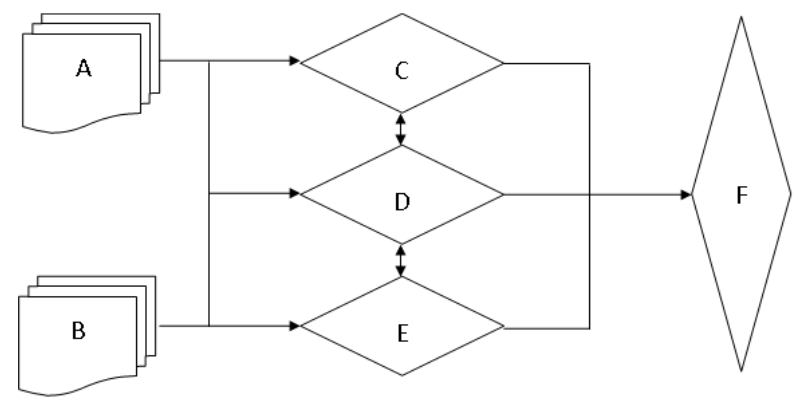

Figure 1. Competitive swimming athletes' technical diagnosis system schematic diagram.

\subsection{Competitive swimming technical optimization control analysis}

Competitive swimming new theory methods study, world competitive swimming technique new development trend recognition extent, swimming technical motion analysis new software, multi-media and others advanced scientific tools mature application and scientific researches as well as in-depth exchange and communicate with coaches, athletes to define national excellent swimming athlete technical optimization target control plan and else four aspects are the basis and foundation of athletes technique optimization control.

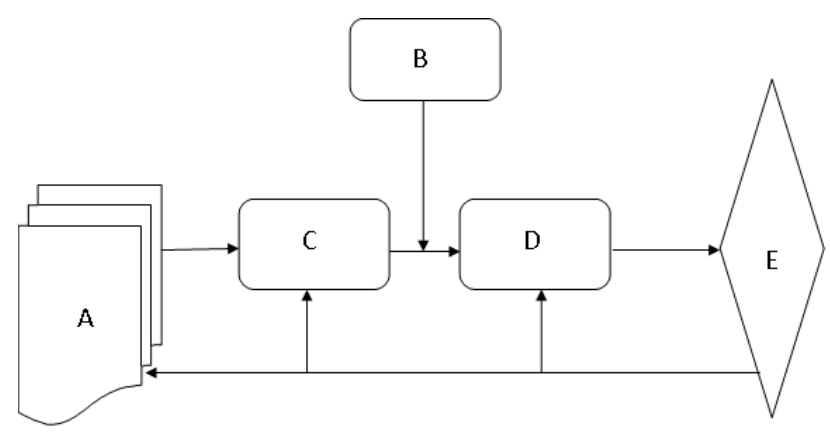

Figure 2. Swimming athlete technique optimization control schematic diagram.

In Figure 2, A: Define national excellent swimming athlete technique optimization target control plan; $\mathrm{B}:$ Scientific researchers assist and participate in coaches teaching and training guidance to athletes, perfection and improvement techniques; C:Timely find out problems in training, make explanation and analysis on spot, let athlete to notice the problems; D:carry on competitive level tracking research on national excellent athletes competitions participation; E: Test preparations' swimming technical training effects and competitive level performance status by competition.

\section{Competitive swimming turning starting techniques}

In Figure 4, we can see that swimming techniques mainly contain starting, turning, on passage swimming, reaching to wall and touching and sprinting so on. Among them, staring and turning techniques occupy crucial position in the whole 
swimming process. Swimming starting and turning rely on legs pedaling and stretching to let human body to get forward initial speed, and improve preliminary speed for athletes underwater sliding. And athlete underwater sliding average speed is up to preliminary speed size to a great extent, which will further affect swimming athlete whole sports performance. To provide reliable theory and technical support for coaches and swimming athletes, it should make research and analysis of excellent swimming athlete starting and turning technical features.

\subsection{Swimming starting technical analysis}

Swimming starting technique refers to the generic terms of methods that swimming athletes use to arrive at fastest speed in shortest time to get rid of static state. It is composed of ready position, takeoff movement, flight phase, entry and sliding and overhand so on six technical links. In current stage researches, in order to avoid troubles, it habitually calls swimming starting ready position to starting front $15 \mathrm{~m}$ such phase as a joint name of swimming starting technique.

Swimming starting postures are ready position before athlete starting, it can ensure athlete to be in the most beneficial starting state, and then get maximum leaving block preliminary speed. With the development of times, swimming competition intense extent increases accordingly, swimming starting postures also has gone through several different development phases. Before the sixties of 20th century, swimming athletes mostly adopted swinging arm style starting postures, since entering into the sixties, grab starting technique dominants, while to nineties of 20thcentury, it appears track starting technique, by far, grab style, forward lean track and back-swing style these three types have become starting postures that world excellent swimming athletes adopt. Now take grab starting posture as an example, analyze it technical movement.

The maximum differences between track staring postures and grab starting postures are difference of takeoff angle and ready position. Adopt track starting posture, due to arms dragging effects, it can accelerated body gravity center forward shifting, by two legs successive exerting, it will get bigger strength, when obtain faster speed, its consumed time will be shorter. And meanwhile track starting postures advantages in perching block time and leaving block instant body initial speed are relative significant.

Time from starting signal to athlete making starting movement is jointly called starting reaction time. In 1985, J.panlo Vilas-boas, M.Joo Cruz and others made researches on Portugal 11 athletes of national team and showed that adopted grab starting athletes perching block time was averagely $0.94 \pm 0.07 \mathrm{~s}$, track style perching block time was averagely $0.90 \pm 0.07 \mathrm{~s}[5]$. Researched on starting reaction time, according to research result, when track style and grab style adopted different starting postures, athletes starting reaction time had significant differences $(\mathrm{P}<0.01)$ track style starting postures were obviously shorter than grab style starting postures reaction time.
Perching block time mainly is composed of starting reaction time and accelerated pedaling and extending time, is generic term that swimming athletes begin to make starting movement to legs leaving block time. Perching block time mainly is up to athlete muscle type, movement proficiency extent and athlete reaction time and else. By far, different scholars have different conclusions on track style and grab style starting postures perching block time long or short. Zhang Ming, Lin Hong etc. carried on mechanical analysis of three kinds of swimming starting postures and found that grab style perching block time was $0.71 \pm 0.01 \mathrm{~s}$, back swing track style perching block time is $0.80 \pm 0.03 \mathrm{~s}$, forward lean track style perching block time is $0.64 \pm 0.02 \mathrm{~s}$, and Tang $\mathrm{Li}$ applied kinematics analysis and research on track style and grab style starting postures, he found that track style perching block time was $0.78 \mathrm{~s}$, which was $0.07 \mathrm{~s}$ more than grab style.

Flight time is directly related to leaving block instant body gravity center initial speed, it is the time from two legs leaving block instant to hand touching water surface. Flight angle being too big or small is bad for the whole swimming process, when flight angle is too big, it will lose horizontal initial speed, and flight angle being too small will shorten flight time that causes body touch water time being earlier, and so will come across underwater resistance, which will generate bad impacts on body flight speed. Research shows, swimming starting flight angle is around $15-20^{\circ}$, and it will more beneficial to swimming starting technique speed performing.

\subsection{Swimming turning technical analysis}

In competitive swimming, 100 meters distance and above 100distances swimming events should make one time or multiple times turning movements, if turning movement well fulfilled, it averagely can save above 0.02 second times, and can save strength, so turning techniques merits affect swimming performance to a certain degree, especially in long distance competitions [8].

In analyzing and researching on free stroke event, due to athlete turns in advance that leads to body to be far from pool wall after turning, causes knee joint angle increases, and leads to leg pedaling and extending effects to be not significant, and meanwhile some athletes cannot keep body horizontally after turning technical movement, body each part is not in the same horizontal line, so that cause body forward direction and force direction are inconsistent, which causes force functions consumption because of scattering and increases body resistance underwater, so that reduces pushoff efficiency after turning. And after athlete flipping, human body and pool wall distance decides pushoff efficiency, after flipping excessive long distance or short distance will cause poor pushoff efficiency, current stage research analysis gets that when swimming athlete thigh and shank included angle as $60^{\circ}$, pushoff efficiency is best.

\section{Conclusions}


Through national swimming team obtained huge achievements in recent years, it fully shows competitive athletes technical diagnosis and optimization can provide strong theory, data and technical support for competitive swimming athlete training method and strategies. It provides scientific and effective practical methods for athlete improving and perfecting technical movements, and be able to let him to faster and better implement self potential maximization. In the paper, it focuses on stating and analyzing competitive swimming's turning and starting technical features and each kind of time factors that affect turning and starting techniques performing. By researching and comparing man and woman different swimming events turning technical experiment data, competitive swimming turning and starting techniques mainly rely on human body legs pedaling and extending movements, so that let human body to get horizontal direction initial speed. And legs strength and explosive power decide athlete leaving wall instant initial speed, which is also key factor in turning and staring techniques that man and woman in different swimming events.

\section{References}

1. X. L. Wu, J. C. Li, Journal of Wuhan Institute of Physical Education, 39, 47-51(2005).

2. W. J. Fan, A. J. Zhou, J. M. Liu, Journal of Beijing Sport University, 25, 847-849(2002).

3. Z. M. Zhang, Journal of Physical Education, 18, 111-114(2011).

4. D. Lin, Journal of Capital College of Physical Education, 23, 540-542, 549(2011).

5. J. H. He, Journal of Wuhan Institute of Physical Education, 41, 63-65(2007).

6. Y. P. Jiang, Journal of Tianjin Institute of Physical Education, 19, 86-87(2004).

7. Y. Lang, Z. H. Liu, H. Y. Liao, Cluster Computing-The Journal Of Networks Software Tools and Applications, 18, 507-516(2015).

8. J. S. Hwang, H. S. Yun, Y. C. Suh, et al. Journal of Coastal Research, 72, 33-38(2014). 